Bol. Soc. Bot. México 46:19-24 (1984).

\title{
Rango de hospedantes de Rhynchosporium secalis
}

\author{
Magda Carvajal Moreno ${ }^{1}$
}

\begin{abstract}
RESUMEN. Se presenta una relación de 75 hospedantes de Rhynchosporium secalis (Oud.) Davis conocidos y reportados en el mundo, así como una lista de los pastos no hospedantes de este hongo y de los hospedantes alternos, con el objeto de ayudar al control cultural de este parásito de la cebada.
\end{abstract}

ABSTRACT. A worlwide list of 75 host plants of Rhynchosporium secalis (Oud.) Davis is given, as well as a list of the grasses that are not host, and of those that are secondary hosts. The aim is to help to achieve agronomic control of this barley parasite.

El rango de hospedantes del hongo $R$. secalis nos ayuda a conocer la forma en que el patógeno sobrevive cuando no hay cultivo y cuáles plantas silvestres, como son los numerosos pastos, actúan como fuentes de inóculo y tendrán que ser eliminados para proteger la cebada. Es importante observar la época del año en que aparece la escaldadura, en diferentes especies de plantas, pues si el cultivo de cebada apenas está germinando y en los pastos silvestres hay lesiones evidentes de escaldadura producidas por $R$. secalis, sí podemos considerar que esos pastos son la fuente de inóculo y no al revés.

Es interesante observar que en muchos casos un patógeno ataca en general a familias de plantas, o sea hospedantes relacionados evolutivamente; en este caso la familia Gramineae es la atacada. Parece ser que el parásito va evolucionando con el hospedante a través de millones de años y cuanto más amplio sea el rango de hospedantes, la relación parasítica es menos estrecha y más reciente. Los parásitos obligados altamente específicos de determinado hospedante han sido seleccionados durante más tiempo y han sufrido aislamiento en cierto momento, lo que ha estrechado la relación parasitaria.

En nuestro caso se observa que, aunque $R$. secalis ataca al trigo (Caldwell, 1937), es más agresivo en la cebada y en el centeno (Kiewnick, 1972).

Este hecho no se puede interpretar como que la cebada y el centeno son evolutivamente más cercanos que la cebada y el trigo, ya que el centeno y el trigo son capaces de cruzarse para dar el triticale y además, desde el punto de vista evolutivo, la cebada está más cerca del género Agropyron (Gould, 1968).

Las relaciones evolutivas de los hospedantes sí existen, pues de hecho son todos de

${ }^{1}$ Departamento de Botánica, Instituto de Biología, Universidad Nacional Autónoma de México. Apdo. Postal 70-233, 04510 México, D.F.

Carvajal-Moreno M. 1984. Rango de hospedantes de Rhynchosporium secalis. Boletín de la Sociedad Botánica de México 46: 19-24. 
la misma familia, pero ya a nivel más específico se encuentran distribuidos en cuatro tribus diferentes.

$R$. secalis ataca por lo menos a 75 hospedantes (tabla 2); sin embargo, es importante señalar de cuáles hospedantes pasa el hongo a la cebada o viceversa, que son los que actuarían como hospedantes alternos del cultivo ya que si sólo pasa de la cebada a ellos no sería de tanta importancia agrícola.

En Lolium multiflorum, Agropyron subsecundum, Phalaris arundinacea y Bromus vulgaris, aparecieron conidios de formas intermedias entre Rhynchosporium secalis y Rhynchosporium orthosporum, además de las típicas (Wilkins, 1973).

El hongo ataca hospedantes a $2500 \mathrm{~m}$ en las montañas de Utah en Bromus carinatus y Bromus inermis (Sprague y Fisher, 1952) y a 2100 m en Nevada, sobre Elymus cinereus (Sprague, 1956).

En Finlandia el hongo esporuló en forma abundante en hojas de centeno y fue poco frecuente en Agropyron repens. Hubo un alto grado de especialización; dos aislamientos de la cebada de primavera y centeno de invierno fueron patogénicos sólo a sus hospedantes originales (Makela, 1974).

Es importante nombrar los pastos que al ser inoculados no presentaron la enfermedad (ver tabla 1).

TABLA 1. Gramíneas que al ser inoculadas con Rhynchosporium secalis no presentaron escaldadura*

\author{
Aegilops truincialis L. \\ Agropyron elongatum L. \\ Avena fatua L. \\ Brachypodium distachyum Beauv. Agrost. \\ Bromus arizonicus (Shear) Stebbins \\ Bromus mollis $\mathrm{L}$. \\ Bromus rubens L. \\ Bromus sterilis L. \\ Cynodon dactylon Steud. \\ Deschampsia danthonioides Munro. \\ Ehrbarta longiflora Sm. Eckl. ex Schult.
}

* Jackson y Webster (1976).

El concepto de rango de hospedantes está íntimamente relacionado con el de raza de patógeno y hay dos puntos de vista opuestos. Por un lado tenemos a Caldwell que separa a cinco razas del hongo con base en los hospedantes atacados y dice que ningún cultivo del hongo infectó a otro hospedante diferente del que provenía (Caldwell, 1931).

Por otro lado, tenemos el punto de vista de Ali y Boyd (1973) y de Schein (1958) que dicen que en la naturaleza el hongo pasa de pastos a la cebada y viceversa.

De modo que si vemos un pasto hospedante cerca de un cultivo de cebada, y el cultivo año tras año baja de producción por escaldadura habrá que considerar poner una variedad de cebada resistente a $R$. secalis y eliminar al pasto.

A continuación se presenta una lista con todos los hospedantes de Rhynchosporium secalis conocidos hasta ahora: 
TABLA 2. Plantas hospedantes de Rhynchosporium secalis con su sitio de registro y su referencia bibliográfica

\begin{tabular}{|c|c|c|}
\hline Planta hospedante & Sitio de registro & Referencia \\
\hline 1. Agropyron ciliaris, Steud. & Japón & 15,2 \\
\hline $\begin{array}{l}\text { 2. Agropyron dasystachyum (Hook) } \\
\text { Scribn. }\end{array}$ & E.U.A. & $6,26,28,29$ \\
\hline 3. Agropyron elmeri Scribn. & E.U.A. & 28,29 \\
\hline $\begin{array}{l}\text { 4. Agropyron intermedium (Host) } \\
\text { Beauv. }\end{array}$ & E.U.A. & 25,31 \\
\hline $\begin{array}{l}\text { 5. Agropyron pungens (Pers.) } \\
\text { Roem. }\end{array}$ & E.U.A. & 24,25 \\
\hline 6. Agropyron repens (L.) Beauv. & $\begin{array}{l}\text { Alemania, Dinamarca, } \\
\text { Gran Bretaña, Finlan- } \\
\text { dia Japón y E.U.A. }\end{array}$ & $2,4,6,8,16,18,19,20,28,29,31$ \\
\hline $\begin{array}{l}\text { 7. Agropyron riparium Scribn. y } \\
\text { Smith. }\end{array}$ & E.U.A. & 25 \\
\hline $\begin{array}{l}\text { 8. Agropyron semicostatum (Steud) } \\
\text { Nees. }\end{array}$ & E.U.A. & 28,29 \\
\hline 9. Agropyron smitbii Rydb. & Argentina, E.U.A. & 22,25 \\
\hline $\begin{array}{l}\text { 10. Agropyron subsecundum (Lk.) } \\
\text { Hitchc. }\end{array}$ & E.U.A. & $28,29,30$ \\
\hline $\begin{array}{l}\text { 11. Agropyron trachycaulum (Link) } \\
\text { Malte (syn. } \\
\text { A. tenerum Vasey). }\end{array}$ & E.U.A. & $6,28,29$ \\
\hline $\begin{array}{l}\text { 12. Agropyron trichophorum (Link) } \\
\text { Richt. }\end{array}$ & E.U.A. & 25 \\
\hline $\begin{array}{l}\text { 13. Agropyron tsukushiense (Honda) } \\
\text { Ohwi var. transiens } \\
\text { (Hackel) Ohwi. }\end{array}$ & Japón & 20 \\
\hline 14. Agrostis alba L. & E.U.A. & 28,29 \\
\hline 15. Agrostis stolonifera L. & Alemania & 2 \\
\hline 16. Avena fatua $\mathrm{L}$. & E.U.A. & 13 \\
\hline 17. Avena sativa $\mathrm{L}$. & Dinamarca & 18 \\
\hline 18. Bouteloua gracilis (H.B.K.) Lag. & E.U.A. & 28,29 \\
\hline 19. Bromus arenarius Labill. & E.U.A. & 6 \\
\hline 20. Bromus arvensis L. & Alemania, E.U.A. & 2,25 \\
\hline 21. Bromus carinatus Hook y Arn. & E.U.A. & $28,29,33,35$ \\
\hline 22. Bromus ciliatus L. & E.U.A. & 34,35 \\
\hline $\begin{array}{l}\text { 23. Bromus frondosus (Shear) Stand } \\
\text { y Wootn. }\end{array}$ & E.U.A. & 28,29 \\
\hline 24. Bromus inermis Leyss. & E.U.A. & $4,9,33,36$ \\
\hline $\begin{array}{l}\text { 25. Bromus lanuginosus Poir } \\
\qquad \begin{array}{l}\text { (probablemente syn. } \\
\text { B. macrostachys } \\
\text { Desf.) }\end{array}\end{array}$ & E.U.A. & 6 \\
\hline 26. Bromus madritensis L. & E.U.A. & 6 \\
\hline 27. Bromus mollis L. & Gran Bretaña & 3,13 \\
\hline 28. Bromus pumpellianus Scribn. & E.U.A. & 28,29 \\
\hline 29. Bromus secalinus L. & E.U.A. & 28,29 \\
\hline 30. Brumus sterilis $\mathrm{L}$. & Gran Bretaña & 3,13 \\
\hline 31. Brumus unioloides H.B.K. & Argentina & 22 \\
\hline $\begin{array}{l}\text { 32. Bromus villosus } \text { Forsk } \\
\qquad \begin{array}{l}\text { (probablemente syn. } \\
\text { de B. rigidus Roth). }\end{array}\end{array}$ & E.U.A. & 6 \\
\hline 33. Bromus vulgaris (Hook) Shear & E.U.A. & 33 \\
\hline 34. Cynosurus cristatus L. & Alemania & 2 \\
\hline
\end{tabular}




Planta hospedante
35. Danthonia californica Boland.
36. Danthonia spicata (L). Beauv.
37. Dactylis glomerata L.
38. Elymus canadensis L.
39. Elymus cinereus Scribn. y Merr
(syn. E. condensatus.
Amer. Auct.)

40. Elymus glaucus Buckl.

41. Elymus innovatus Beal.

42. Elymus junceus Fisch.

43. Elymus triticoides Buckl.

44. Elymus virginicus L.

45. Elymus robustus Scribn.

46. Elymus smithii

$$
\text { y J.G. Sm. }
$$

47. Elymus striatus

48. Elymus villosus Muhl.

(probablemente syn. de $E$. striatus).

Autores americanos no Willd.

49. Festuca elatior L.

50. Festuca idahoensis Elmer.

51. Holcus lanatus L.

52. Hordeum brachyantherum Nevski.

53. Hordeum distichum L.

54. Hordeum jubatum L.

55. Hordeum leporinum Link.

56. Hordeum murinum L.

57. Hordeum nodosum L.

(probablemente syn.

H. brachyantherum

Nevski).

58. Hordeum pusillum Nutt.

59. Hordeum sativum L.

60. Hordeum spontaneum L.

61. Hordeum vulgare L.

62. Lolium multiflorum Lam. syn. Lolium italicum

$$
\text { A. Br.) }
$$

63. Lolium perenne $\mathrm{L}$.

64. Lolium rigidum Gaud.

65. Milium effusum L.

66. Phalaris arundinacea $\mathrm{L}$.

67. Phleum pratense $\mathrm{L}$.

68. Poa compressa $\mathrm{L}$.

69. Poa memoralis $\mathrm{L}$.

70. Poa pratensis $\mathrm{L}$.

71. Poa trivialis L.

72. Secale cereale L.

73. Secale montanum Guss.

\section{Sitio de registro Referencia}

E.U.A.

E.U.A.

5

E.U.A., Gran Bretaña

$11,28,29$

Argentina, E.U.A.

E.U.A.

$3,6,9,26,37$

$5,22,28,29$

32

E.U.A.

E.U.A.

$26,28,29,32,33$

E.U.A.

28,29

E.U.A.

28,29

E.U.A.

E.U.A.

$13,28,29$

$6,28,29$

5

Argentina 22

E.U.A. $\quad 24$

E.U.A.

6

Argentina 22

E.U.A. 25

Alemania 2

E.U.A. $\quad 34,35$

E.U.A. $\quad 6,28,29$

E.U.A. $\quad 6,28,29$

Australia 1

Alemania, Gran Breta- $\quad 2,16,21,26,28,29$

ña, E.U.A.

E.U.A.

6

E.U.A.

Gran Bretaña 37

Australia 1

E.U.A., Gran Bretaña $\quad 6,13,16,17,28,29$

Alemania, Gran $\quad 2,16,20,21,28,29,37$

Bretaña, Japón,

E.U.A.

Alemania, Australia, $\quad 1,2,26,28,29,37$

Gran Bretaña, E.U.A.

Australia 1

Dinamarca 18

E.U.A. $\quad 28,29$

Alemania 2

Alemania 2

Alemania 2

Alemania 2

Alemania 2

Gran Bretaña, Japón, $\quad 6,14,16,17,19,28,29$

E.U.A., Finlandia

E.U.A.
(28)

\section{2}

2

28,29 


\begin{tabular}{lll}
\hline \multicolumn{1}{c}{ Planta hospedante } & Sitio de registro & Referencia \\
\hline 74. Triticum aestivum L. & Alemania, E.U.A. & $6,7,12$ \\
75. Triticum vulgare L. Vill. & Australia, Alemania, & $1,6,7,12$ \\
& E.U.A. & \\
\hline
\end{tabular}

Los números de las referencias de la tabla 2 corresponden a los siguientes autores: (1) Ali y Boyd, 1973; (2) Bartels, 1928; (3) Brooks, 1928; (4) Caldwell, 1929; (5) Caldwell, 1931; (6) Caldwell, 1937; (7) Davis, 1919; (8) Davis, 1922; (9) Dreschler, 1921; (10) Gould, 1968; (11) Haskell, 1926; (12) Heinsen, 1901; (13) Jackson y Webster, 1976; (14) Kajikawa, 1968; (15) Kajiwara e Iwata, 1963; (16) Kay y Owen, 1973; (17) Kiewnick, 1972; (18) Lind, 1913; (19) Makela, 1974; (20) Nishihara, 1979; (21) Owen, 1958; (22) Sarasola y Campi, 1947; (23) Secretaría de Agricultura y Recursos Hidráulicos, 1982; (24) Schein, 1958; (25) Schein, 1960; (26) Sprague, 1935; (27) Sprague, 1946; (28) Sprague, 1950 a; (29) Sprague, 1950 b; (30) Sprague, 1954; (31) Sprague, 1955; (32) Sprague, 1956; (33) Sprague, 1958; (34) Sprague, 1960; (35) Sprague, 1962; (36) Sprague y Fisher, 1952 y por último (37) Wilkins, 1973.

\section{CONCL_USIÓN}

Rhynchosporium secalis ataca a 75 plantas hospedantes conocidas de cuatro diferentes tribus de la familia Gramineae, de modo que cuando se encuentren creciendo cerca de un cultivo de cebada, representarán un peligro, ya que serán los hospedantes alternantes de este hongo y habrá más posibilidades de que la escaldadura de la cebada sea más agresiva al aumentar la cantidad de inóculo, y difícil de erradicar, ya que aunque se coseche la cebada, el hongo persiste en su hospedante alternante presente en ese momento.

\section{LITERATURA CITADA}

Ali, S.M. y W.J.R. BoyD. 1973. Host range and physiological specialization in Rhynchosporium secalis. Aust. J. Agric. Res. 25:21-31.

Bartels, F. 1928. Studien über Marssonina graminicola. Forschm. Geb. Pflkrankh., Berl. 5:73-114.

Вrooks, F.T. 1928. Observations on Rhynchosporium secalis (Oud) Davis, leaf blotch of barley and rye. New Pbytol. 27:215-219.

CALDWELl, R.M. 1929. Preliminary results from cross inoculation and culture studies upon the fungus Rhynchosporium secalis (Oud.) Davis causing scald of cereals and other grasses. (Abstract). Phytopathology 19:104.

1931. Specialization and parasitism of the genus Rhynchosporium. Abstract. Phytopathology 21:109-110. 1937. Rhynchosporium scald of barley, rye and some other grasses. J. Agric. Res. 55:175-198.

DAvIS, J.J. 1919. Notes on parasitic fungi in Wisconsin. VI. Wisconsin Acad. Sci. Arts. Letters, Trans. 19:705-727. 1922. Notes on parasitic fungi in Wisconsin. VIII. Wisconsin Acad. Sci. Arts. Letters, Trans, 20:413-431.

DresCHLER, C. 1921. Ocurrence of Rhynchosporium on Dactylis glomerata and Bromus inermis. Phytopathology 11(1):42.

Gould, F.W. 1968. Grass systematics. McGraw-Hill Book Company. USA. 367 p.

Haskell, R.J. 1926. Scald caused by Rhynchosporium secalis (Oud.) Davis. U.S. Bur. Plant Indus. Plant Disease Reporter Suppl. 48:341.

HeInsEn, E. 1901. Beobachtungen über den neunen Getreoidepilze Rhynchosporium graminicola. Jabrb. Hamburg Wiss. Anst. 18:43-45.

JACKSON, L.F. y R.K. WEBSTER. 1976. Seed and grasses as possible host sources of Rhynchosporium secalis for barley in California. Plant Disease Reporter 60:233-236. 
KAJIKAWA, T. 1968. Comparative studies on the pathogenicity of barley-M and rye-M scald fungus Rhynchosporium secalis. Bull. Nat. Inst. Agric. Sci., Ser. C (22):259-268.

. Y. Iwata. 1963. Studies on the strains of the barley scald fungus, $R$. secalis (Oud.) Davis. Bull. Natl. Inst. Agric. Sci. Tokyo, Series C (Plant Patbol. Entomol). Japan 15:1-82.

KAY, J.G. y H. Owen. 1973. Host range of Rhynchosporium secalis Barley. Brit. Mycol. Soc. Trans. 60:413-422.

KIEWNICK, L. 1972. A little-noticed leaf spot disease of barley and rye Rhynchosporium secalis. Gesunde Pflanzen. 24:139-140.

LIND, J. 1913. Danish fungi as represented in the herbarium of E. Rostrup. Copenhagen. 648 p.

MAKELA, K. 1974. Ocurrence of Rhynchosporium secalis (Oud.) J.J. Davis on spring barley and winter rye in Finland. Agric. Soc. Finland. 46:103-117.

NishiHara, N. 1979. Rhynchosporium scald of Lolium multiflorum and Agropyron repens in Japan. Bull. Nat. Grassl. Res. Inst. 15:103-115.

OwEN, H. 1958. Physiologic specialization in R. secalis. Trans. Br. Mycol. Soc. 41:99-108.

SARASOlA, J.A. y M.D. CAMPI. 1947. Reacción de algunas cebadas con respecto a Rhynchosporium secalis en Argentina. Rev. Invest. Agric. Buenos Aires 1:243-260.

Secretaría de Agricultura y Recursos Hidráulicos. 1982. Notisabr. 3. Marzo: 11.

SCHEIN, R.D. 1958. Pathogenic specialization in Rhynchosporium secalis. Phytopathology 48:477-480. 1960. Physiologic and pathogenic specialization of R. secalis. Penn. State Univ. Agric. Exp. Stn. Bull. 664:1-29.

SPRAgue, R. 1935. A preliminary checklist of the parasitic fungi in cereals and other grasses in Oregon. Plant Diease Reporter 19:156-186.

1946. Rootrots and leafspots of grains and grasses in the Northern Great Plains and Western States. U.S.D.A. Bur. Pl. Ind. Plant Disease Reporter Suppl. 163:167.

1950 (a). Diseases of cereals and grasses in North America. Ronald Press Co., Nueva York. 421-423.

1950 (b). Some leafspot fungi on Western Gramineae. V. Mycologia 42:758-768.

1954. Some leafspot fungi on Western Gramineae. VII. Mycologia 46:85.

1955. Some leafspot fungi on Western Gramineae. IX. Mycologia 47:835-845.

1956. Some leafspot fungi on Western Gramineae. X. Mycologia 48:749.

1958. Some leafspot fungi on Western Gramineae. XX. Mycologia 50:822.

1960. Some leafspot fungi on Western Gramineae. XIII. Mycologia 52:372.

1962. Some leafspot fungi on Western Gramineae. XVI. Mycologia 54:55, 605.

y G.W. FisHeR. 1952. Check list of the diseases of grasses and cereals in the Western U.S.A. and Alaska. Wash. Agric. Exp. Sta. Circ. 194.

WILKINS, P. 1973. Infection of Lolium multiflorum with Rhynchosporium species. Plant Pathology 22:107-111. 